\title{
Joint optimization of operation and maintenance policies for solar-powered Microgrids
}

\author{
Khashayar Mahani ${ }^{1}$, Zhenglin Liang ${ }^{1}$, Ajith Kumar Parlikad and Mohsen A. Jafari, Member, IEEE ${ }^{2}$
}

\begin{abstract}
In a solar-powered microgrid (MG), the optimal maintenance strategy is influenced by the downtime cost of the photovoltaic (PV) system, which in turn depends on the operation PV within the MG network. Also, the dispatch policy used in the MG will influence the economic feasibility of maintenance plans. In this paper, we present an approach for optimizing the operation and maintenance policy jointly for a solar-powered MG considering the dependence between the two policies. The two-layered approach presented in this paper seeks to unify the practicality of simulation and the efficiency of analytical models. In the upper layer, we optimize the operation of MG by solving the optimal power dispatch within the MG network using linear programming approach. Then, we calculate the penalty costs under the aging conditions of PV systems. In the bottom layer, by incorporating the penalty costs as input parameters, we use a continuous-time Markov chain model to calculate the optimal maintenance policy for the PV system. The proposed approach could be used in the stipulation process between MG owner and PV system maintenance provider to minimize the money waste on both sides.
\end{abstract}

Index Terms-Two-level optimization, operation dependence, condition-based maintenance, linear programming and continuous-time Markov chain

\section{NOMENCLATURE}

\begin{tabular}{|c|c|c|}
\hline$d$ & Index of day & $1 / \mu_{\mathrm{c} l}^{(\alpha)}$ \\
\hline . & Index of time interval & \\
\hline$k$ & Index of renewable node & \\
\hline$s$ & Index of energy storage unit & $1 / \lambda_{\mathrm{F}}$ \\
\hline$l$ & Index of demand node & \\
\hline$g$ & Main power grid & $\mathrm{C}_{\text {in }}^{\prime}$ \\
\hline$C A P_{S}$ & Storage $s$ energy capacity (kWh) & \\
\hline$P_{s}$ & Energy storage rated capacity $(\mathrm{kW})$ & $1 / \lambda_{i n}^{(\alpha)}$ \\
\hline$L_{d}(l, t)$ & $\begin{array}{l}\text { Total demand during time interval } t \text { at node } l \\
\text { in day } d\end{array}$ & $1 / \mu_{\text {in }}$ \\
\hline$R_{d}(k, t)$ & $\begin{array}{l}\text { Total generation during time interval } t \text { at } \\
\text { renewable node } k \text { in scenario } s c\end{array}$ & \\
\hline$e_{g, s}(d, t)$ & $\begin{array}{l}\text { Total energy charged from the grid during } t \text { in } \\
\text { storage unit } s \text { in day } d\end{array}$ & $1 / \mu_{\mathrm{M}}$ \\
\hline$e_{k, s}(d, t)$ & $\begin{array}{l}\text { Total energy charged from renewable node } k \\
\text { during } t \text { in storage unit } s \text { in day } d\end{array}$ & $C_{D}^{\prime(\alpha)}$ \\
\hline$e_{s, l}(d, t)$ & $\begin{array}{l}\text { Total energy discharged during } t \text { from storage } \\
s \text { to demand node } l \text { in day } d\end{array}$ & $1 / \mu_{\mathrm{R}}$ \\
\hline
\end{tabular}

\footnotetext{
${ }^{1} \mathrm{~K}$. Mahani and Z. Liang contributed equally to the work K. Mahani and M. A. Jafari are with the Department of Industrial and Systems Engineering, Rutgers University, Piscataway, NJ 08854 USA (e-mail: km723@scarletmail.rutgers.edu, jafari@ rci.rutgers.edu).
}

$e_{g, l}(d, t) \quad$ Total energy from the grid during time interval $t$ to demand node $l$ in day $d$

$e_{k, l}(d, t) \quad$ Total energy from renewable $k$ during $t$ to demand node $l$ in day $d$

$\eta_{s} \quad$ Energy storage $s$ one-way efficiency

$E P_{d}(t) \quad$ Electricity price in time interval $t$ for day $d$

$S O C_{s}(d, t) \quad$ Storage $s$ energy level $(\mathrm{kWh})$ at the end of time interval $t$ in day $d$

$S F \quad$ Safety reserve capacity for energy storage unit

$e^{e s l_{s, l}}(t, d) \quad$ Storage "s"-Demand "l" eligibility number (day " $d$ " - time interval "t"), binary

$\operatorname{ers}_{k, s}(t, d) \quad$ Renewable "k"-Storage "s" eligibility number (day $d$ - time interval " $\mathrm{t}$ "), binary

$\operatorname{erl}_{k, l}(t, d) \quad$ Renewable " $\mathrm{k}$ "-Demand "l" eligibility number (day $d$ - time interval " $t$ "), binary

$D O C_{d} \quad$ Optimal daily operation cost in day $d$

$b^{(\alpha)} \quad$ The threshold of major maintenance activity for the $\alpha^{\text {th }}$ photovoltaic system

$m^{(\alpha)} \quad$ The number of degradation states of the $\alpha^{\text {th }}$ photovoltaic system

$n^{(\alpha)} \quad$ The number of failure sudden modes of the $\alpha^{\text {th }}$ photovoltaic system

$\lambda_{m}^{(\alpha)}$

$C_{s, l}^{\prime(\alpha)}$

The deterioration rate for the $\alpha^{\text {th }}$ photovoltaic system $\alpha$ at state $m$

Cost for each corrective maintenance after mode $l$ sudden failures on the $\alpha^{\text {th }}$ photovoltaic system

Duration of corrective maintenance after mode $l$ sudden failures on the $\alpha^{\text {th }}$ photovoltaic system

Mean time between two successive mode $l$ sudden failures on the $\alpha^{\text {th }}$ photovoltaic system Cost for each inspection of the $\alpha^{\text {th }}$ photovoltaic system

Mean time between two successive inspections on the $\alpha^{\text {th }}$ photovoltaic system

Mean duration of inspection on photovoltaics $\alpha$

Cost for each major maintenance activity of the $\alpha^{\text {th }}$ photovoltaic system

Mean duration of major preventive maintenance on the $\alpha^{\text {th }}$ photovoltaic system Cost for each replacement activity of the $\alpha^{\text {th }}$ photovoltaic system

Mean duration of replacement on the $\alpha^{\text {th }}$ photovoltaic system 
$C_{p}^{(\alpha)} \quad$ Planned per unit downtime cost for the $\alpha^{\text {th }}$ photovoltaic system

$C_{u}^{(\alpha)} \quad$ Unplanned per unit downtime cost for the $\alpha^{\text {th }}$ photovoltaic system

$C_{v, i}^{(\alpha)} \quad$ Penalty caused by the performance degradation of the $\alpha^{\text {th }}$ photovoltaic system.

$\pi_{i, j}^{(\alpha)}$

$C_{S}^{(\alpha)}$

$C_{G}$ Probability of the $\alpha^{\text {th }}$ photovoltaic system being in state $(i, j)$

Time-averaged operating cost of the $\alpha^{\text {th }}$ photovoltaic system

The overall expected operational and maintenance cost for the microgrid

\section{INTRODUCTION}

$\mathrm{M}$ icrogrids (MGs) are small-scale power networks composed of multiple energy resources and, in some cases, distributed energy storage devices (ESDs). They are seen to be increasingly important to achieve a reliable, flexible, and sustainable electricity network. In this paper, we focus on two aspects that influence the cost-effectiveness of microgrids - the operation control and maintenance policies - and the relationship between them. In particular, we examine the significance of ESDs on the policies and hence the overall operational cost of the MG. In such type of MGs, ESDs play a role of storing energy when surplus energy is produced and discharging to support demands when needed. Due to the uncertain nature of the power generation by renewable sources [1] and demand profiles within the MG, it poses a challenge on managing the operation of MGs. To overcome this challenge, the related advancement has been achieved on supporting MG owners to decide whether or not to use ESDs, optimizing the size of ESDs $[3,4,5]$, and scheduling the charge and discharge times for these ESDs [6,17].

In general, ESDs could improve the reliability and power quality of a MG. Moreover, it is capable of providing an economic benefit in a deregulated energy market [7]. It encourages utility company to shift and shave peak load [6]. In the light of this, the operation and control of a MG need to be taken into account the power flow between entities within the $\mathrm{MG}$, as well as the power flow between MG and main grid. Khalilpour and Vassallo [2] developed a decision support tool for scheduling of PV-battery systems based on a detailed power flow model. Cost saving through simultaneously managing energy production and demand is another aspect that has been focused on [8]. The latest development in this area enables a near-real-time optimal charge and discharge control policies for a MG with multiple ESDs [9].

Maintenance is also an important issue in MGs, which may have a major impact on the overall ownership costs of the grid. As studied by [10], good maintenance and inspection policies are essential for improving the financial viability of the MG. A particular focus in the area is to examine the safety hazards [11], failure and performance deterioration [12] of photovoltaic (PV) systems in MGs. Hence, an online monitoring system may appear beneficial as it may improve the maintenance performance of PV systems within a MG and in turn increase the profit of the MG. In [13], authors developed a continuoustime Markov chain model for PV systems that are subject to deterioration and failure. The study had shown implementing condition monitoring is more favorable for both MG owner and maintenance provider by comparing with manual inspections. In a MG, maintenance policies that control the availability of PV systems can subsequently influence the energy generation and operation policy of the MG. Moreover, an effective energy storage policy can reduce the downtime penalty cost, if the stored energy can be used to satisfy demand during the downtime of PV systems caused by preventive maintenance or failure. However, the interplay/dependence between operation policy and maintenance policy is still underexplored in the context of the microgrid. In this paper, we refer such type of dependence between operation and maintenance as "operation dependence". The novelty and contribution of our paper are it consists of following five aspects collectively.

1. It is a two-layered approach that includes an upper layer for simulating the operation of $\mathrm{MG}$ and a lower layer for modeling the deterioration and maintenance of PV systems. Through such layer separation, the mathematical tractability of the lower layer is preserved.

2. In the upper layer, we formulate the operation of a MG as an optimal dispatch problem. The discharging and charging of ESDs are optimized in a way to maximize the value of MG. The model formualtes the power flow of the MG with details. Moreover, the model is capable of integrating historical data on demand profiles, solar radiation, and electricity price, which indicates a good applicability in practice.

3. In the lower layer, the deterioration and maintenance of the PV systems are formulated by continuous-time Markov chain. Both the performance degradation caused by the malfunction of PV arrays and invertor failure are considered. Also, the model considers the maintenance duration.

4. We have applied our approach on a practical MG to test the practicality. The value of ESDs is demonstrated from operation and maintenance perspectives through a comparative study.

5. Finally, our study could provide insights for both maintenance service providers and MG owners. A warranty contract that based on the performance of PV systems could be mutually beneficial for both sides compared with a fixed amount warranty contact. Our operation and maintenance model can support both sides to this end.

The rest of paper is structured as follows: In section II, we introduce the general set-up of the MG and the mechanism for failure and performance degradation for the PV systems within the MG. Section III describes the modeling approach to optimize the operation and maintenance of the MG. Section IV validates the approach by applying it to a practical solarpowered MG in the US. The optimal operation and maintenance strategies are demonstrated. Moreover, an analysis is provided on the value of ESDs in this context. Finally, section $V$ presents the concluding remarks of the paper.

\section{SYSTEM DESCRIPTION}

We consider a grid-connected community level MG, with PV resources as the source of power as illustrated in Fig 1. The PV output may differ from the system load from time to time. When the PV output is greater than the load, the ESDs absorb this 
excessive power. Hence, the energy charged from PV resources during off-peak hours can be utilized during peak hours to shave the peak demand.

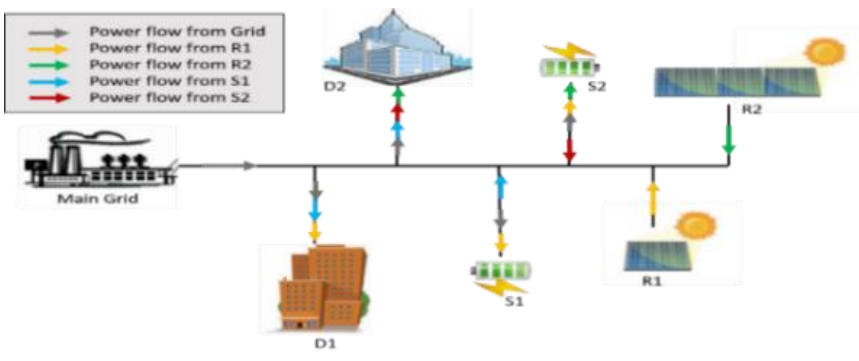

Fig 1: An illustrative example of a $M G$ configuration

The demands of the community are primarily satisfied by the power generated on-site by the PV systems and ESDs within the grid. Alternatively, the main grid can also supply power to the community. In this case, the operation cost of the MG is the expenditure on purchasing electricity from the main grid to supplement and satisfy the electricity demands in the community. We assume that the owner of the MG participates in the wholesale day-ahead market. Due to the cost of buying electricity from the main grid is varying throughout the day, the total operation cost can be reduced by optimizing the charging and discharging time of ESDs. In our approach, the operation policy depends on the demand level, on-site generated power, electricity price as well as the performance and availability of PV systems.

The PV system is configured in multiple arrays. As illustrated in Fig. 2 multiple PV modules are serially connected within each array.

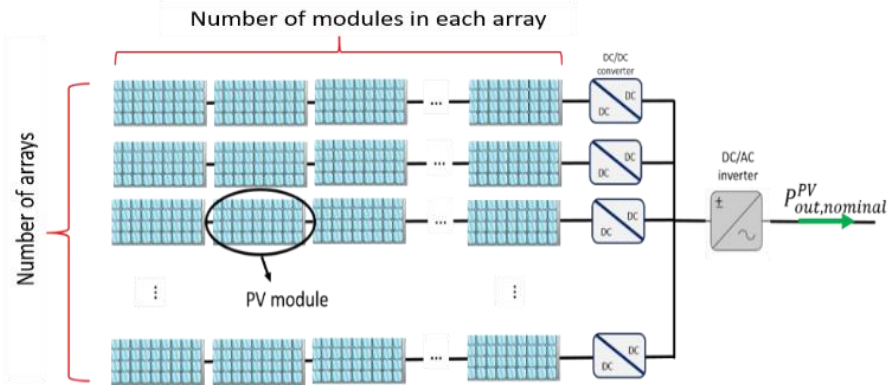

Fig. 2: Configuration of PV systems

The failure of a PV module will stop its array from operating. Thus, despite the low failure rate of PV modules, the failure rate of serially connected PV arrays is still non-negligible [19, 20]. The energy generation capability of the PV system is proportional to the number of functional arrays. Consequently, the failure of a PV module will result in performance degradation of the PV system. In new system, the PV module may also be bypassed by diodes due to an open failure or shading effect. The bypass of PV module generally could lower the output of a string, rather than causing an outage of the string. Even though the proposed maintenance model is capable to deal with such system, in this study, we do not consider the bypass of modules [20]. All PV arrays are connected to a DC/AC inverter. The inverter is used to convert the electricity generated by the PV system to the regulated AC voltage. The failure of the inverter will immediately disconnect the PV system from the MG. Such type of failure is formulated as a sudden failure in our designed maintenance model. The unavailability or performance degradation of PV systems will affect the operational decision of ESDs. We assume that the performance of PV systems can be observed and analyzed by grid operator continuously.

The objective of operation policy is to determine the optimal power dispatch among different nodes within the MG, according to the performance level and availability of PV systems. Taking into account the operation dependence, the objective of maintenance policy is to identify the optimal maintenance threshold (a degradation threshold triggering replacement of the failed PV modules) for the PV systems so that the expected annual ownership cost (operation cost and maintenance cost) of the MG is minimized.

\section{MODELING APPROACH}

Our modeling approach contains two layers. The upper layer aims to optimize the operation of the MG under different types of operation constraints by optimally charge/discharge ESDs. The output of this model is the operation cost of MG under different conditions of PV systems. This output then forms a part of the input to the lower layer, which aims to optimize the maintenance policies for the PV systems in the long term. A holistic view of the top-down approach is illustrated in Fig. 3.

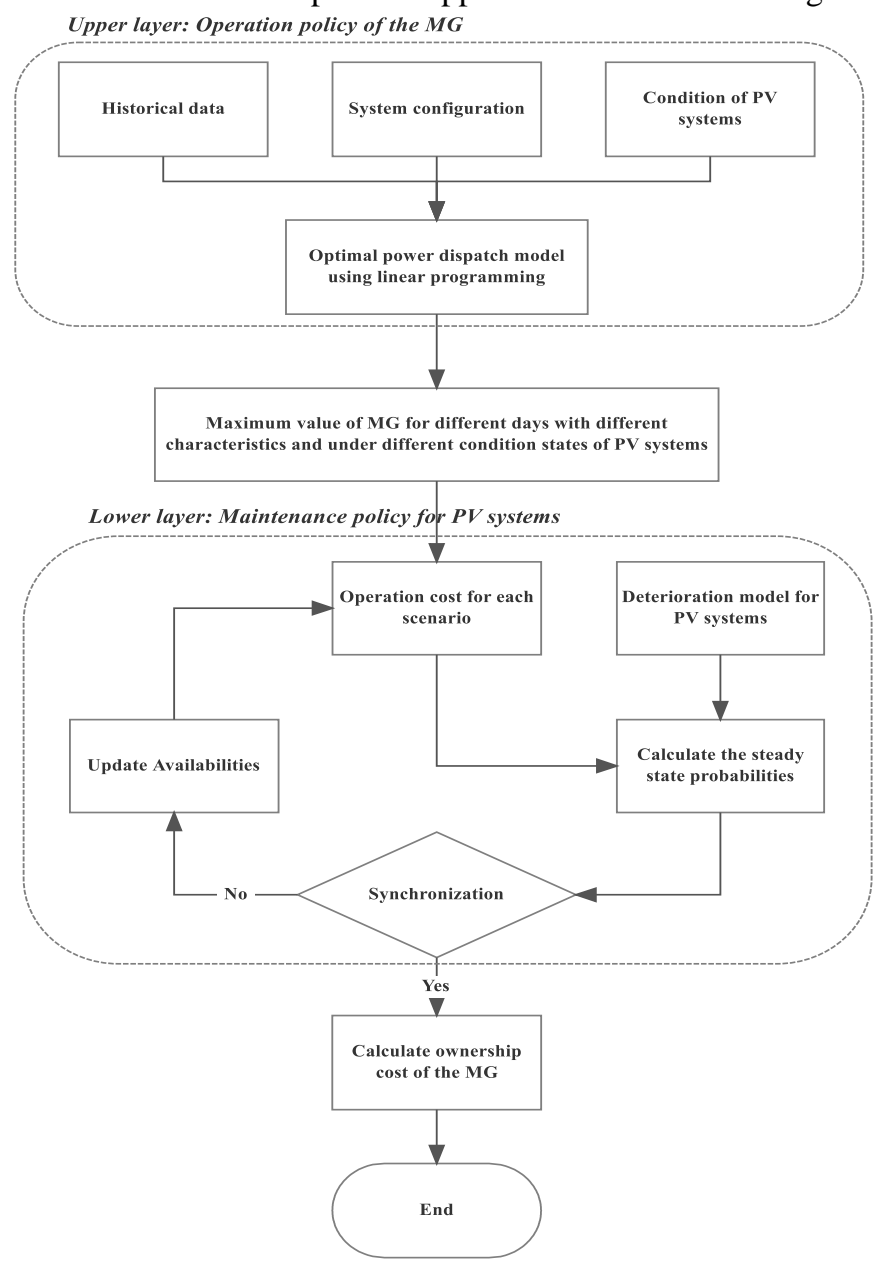

Fig. 3: Schematic diagram of the top-down approach

In the upper layer model, we compute the optimal power dispatch problem using linear programming under different 
condition states of the PV systems and for each individual day based on the historical data. Days are distinguished by three stochastic variables, namely electricity demand, solar radiation, and electricity price. Three years' historical data (available on PJM website) have been used to characterize hourly profiles of demand, electricity price and solar radiation each day. The operation model optimizes the amount of charged and discharged energy (as decision variables) of ESDs during the different time intervals for each individual day. This optimal solution also depends on the state of network elements. such as the degradation state (condition) of PV systems and the network configuration (connectivity of different nodes). The output from operation model is the lower bound for the microgrid operation cost for each individual day existed in historical dataset under different conditions of PV systems. By comparing the operation cost of the MG in the good condition state of PV systems (100\% performance) with any individual degraded state (or failure state) of the PV system, we can calculate the penalty cost due by performance degradation (or failure) of $\mathrm{PV}$ systems. This information is used to formulate of maintenance policy of PV systems. In this way, we link the operation policy and the maintenance policy of the MG. In the lower layer, we consider the situation where the maintenance policy of one PV system changes its availability and may in turn influence the downtime penalty cost of other PV systems and sequentially affect the optimization of maintenance policies. We use an iterative approach to synchronize the maintenance policies of PV systems so that they can reach the optimal solution simultaneously. The final output of the model is the optimal ownership cost of the MG. In the next subsections, we will describe the formulation of the upper and lower layer models.

\section{A. Upper layer (system operation model)}

The objective of the upper layer is to minimize the operation cost of the MG by adjusting the charging and discharging of ESDs based on the scenario and performance of PV systems. We apply the linear programming to optimize the operation of the MG for each scenario. The detail of the objective function and different types of operational constraints of the MG will be explained with more details in equations (1) and (2)-(8) respectively.

Objective function: The daily operation is optimized for each scenario. A scenario contains the information of the electricity demand, generation profile of PV systems and electricity price profile in the given day "d". The objective function then expresses as (1):

$$
\min \left\{\sum_{t}\left[E P_{d}(t)\left(\sum_{l} e_{g, l}(d, t)+\sum_{s} e_{g, s}(d, t)\right)\right]\right\}
$$

The decision variables are the amount of energy charge and discharge by an ESD in a unit time (hour). Note that we assume the voltages of different nodes are maintained in the feasible region. The objective function is to minimize the overall expenditure on purchasing electricity from the main grid. The purchased electricity is used to either charge storages $\left(e_{g, s}\right)$ or supply demands $\left(e_{g, l}\right)$. The minimization process is subject to multiple types of constraints, which are listed as below:

Storage operation constraints: In each scenario, the total amount of inflow and outflow electricity for each storage node in each time interval is limited to its rated power capacity.

$$
e_{g, s}(d, t)+\sum_{k} e_{k, s}(d, t)+\sum_{l} e_{s, l}(d, t) \leq P_{s}, \forall s, t, d
$$

As illustrated in (2), multiple charging and discharging actions are allowable during each hour. However, the summation of inflow and outflow is limited by the rated capacity of the storage unit. The storage level at a given time interval is calculated by the storage level at the previous time interval and the charging and discharging energy during the time interval.

$$
\begin{gathered}
S O C_{s, t, d}=S O C_{s, t-1, d}+\eta_{s} \times\left(e_{g, s}(d, t)+\right. \\
\left.\sum_{k} e_{k, s}(d, t)\right)-\frac{\sum_{l} e_{s, l}(d, t)}{\eta_{s}}, \forall s, t, d
\end{gathered}
$$

We assume that at the beginning of the day storage level is at the $50 \%$ of maximum capacity and it has to reach to the same level at the end of the day. It is intuitive that the storage level cannot exceed the maximum capacity of the $\operatorname{ESD}\left(C A P_{S}\right)$ and cannot reduce below the safety reserve capacity $\left(S F_{S}\right)$.

$$
S F_{s} \times C A P_{s} \leq S O C_{s, t, d} \leq C A P_{s}, \forall s, t, d
$$

On-site renewable resource constraint: Electricity generated by a renewable unit is used to serve demand nodes and charge the storage nodes which are connected to it.

$$
R_{d}(k, t) \geq \sum_{l} e_{k, l}(d, t)+\sum_{s} e_{k, s}(d, t), \forall k, t, d
$$

Demand constraint: Electricity load at each demand node has to be satisfied. The portion of demands is satisfied by on-site generation and discharged electricity from storages, and the remain has to be satisfied by purchasing from the main grid.

$$
\begin{aligned}
L_{d}(l, t)= & \sum_{k} e_{k, l}(d, t)+\sum_{s} e_{s, l}(d, t)+ \\
& e_{g, l}(d, t), \forall l, t, d
\end{aligned}
$$

Configuration and availability constraints: The configuration of the MG is defined by three binary matrices (ESL, ERL, and ERS). The value 1 indicates the two nodes are connected, and 0 indicates no connection. Sometimes, assets within the MG may become unavailable. We use a binary number $\operatorname{erl}_{k, l}(t, d)$ to indicate the connection between $k^{\text {th }} \mathrm{PV}$ system and $l^{\text {th }}$ demand node at time " $\mathrm{t}$ " in day "d".

$$
\begin{array}{rlrl}
0 \leq e_{k, l}(d, t) \leq M \times \operatorname{erl}_{k, l}(t, d), & & \forall l, k, t, d \\
0 \leq e_{s, l}(d, t) & \leq M \times \operatorname{esl}_{s, l}(t, d), & & \forall l, s, t, d \\
0 \leq e_{k, s}(d, t) & \leq M \times \operatorname{ers}_{k, s}(t, d), & & \forall s, k, t, d
\end{array}
$$

where "M" is a very big number (e.g. 10 million). More details about the optimal operation and control of this network could be found in [9].

For given input profiles and performance of PV systems, the operation of the MG can be optimized. We refer the optimized daily cost under given day "d" and performance of PV systems as $\operatorname{DOC}\left(\mathrm{d}, X^{(1)}, \ldots, X^{(k)}\right) . X^{(\alpha)}$ is a random variable that indicates the performance of $\alpha^{\text {th }}$ PV system. For a PV system with $m_{\alpha}$ number of arrays, $X_{m_{\alpha}}^{(\alpha)}$ indicates all arrays are functional. $X_{i_{\alpha}}^{(\alpha)}$ indicates $i_{\alpha}\left(i_{\alpha}<m_{\alpha}\right)$ number of arrays are functional. Therefore, we have $X^{(\alpha)}=$ $\left\{X_{m_{\alpha}}^{(\alpha)}, \ldots, X_{i_{\alpha}}^{(\alpha)}, \ldots, X_{0_{\alpha}}^{(\alpha)}\right\}$. Let $\overline{D O C^{*}}$ indicates the expected daily cost over all existed days in the historical data set when the PV system amongst the MG is ideal. We signified the overall number of days as $N_{d}$. Then $\overline{D O C^{*}}$ can be expressed as:

$$
\overline{D O C^{*}}=\frac{\sum_{d=1}^{N_{d} D O C}\left(d, X_{\left.m_{1}, \ldots, X_{m_{k}}^{(k)}\right)}^{(k)}\right.}{N_{d}}
$$


We assume the planned preventive maintenance can be scheduled when the impact on the operation of the MG is minimized. $\overline{D O C_{p}^{(\alpha)}}$ is the expected the operation cost when $\alpha^{\text {th }}$ PV system is offline due to preventive maintenance.

$\overline{D_{O O C}^{(\alpha)}}=$

$\min _{d}\left[\operatorname{DOC}\left(\mathrm{d}, X_{0_{\alpha}}^{(\alpha)}, \mathbb{E}\left[X^{(1)}, \ldots, X^{(\alpha-1)}, X^{(\alpha+1)}, \ldots, X^{(k)}\right]\right)\right]$

$\mathbb{E}\left[X^{(1)}, \ldots, X^{(\alpha-1)}, X^{(\alpha+1)}, \ldots, X^{(k)}\right]$ is interrelated with the maintenance strategy of PV systems. It is computed with iteration. To initialize, we assign equal probability for all $X^{(1)}, \ldots, X^{(k)}$. Therefore, the equation (12) is equal to as

$$
\overline{D O C_{p}^{(\alpha)}}=\frac{\min _{d}\left[\sum \operatorname{DOC}\left(\mathrm{d}, X_{0_{\alpha}}^{(\alpha)}, X^{(1)}, \ldots, X^{(\alpha-1)}, X^{(\alpha+1)}, \ldots, X^{(k)}\right)\right]}{\prod_{i=1}^{\alpha-1} \overline{\overline{X^{(l)}}} \prod_{i=\alpha+1}^{k} \overline{\overline{X^{(l)}}}}
$$

where $\overline{\overline{X^{(l)}}}$ indicates the cardinality of $X^{(i)} \cdot \overline{D O C_{u}^{(\alpha)}}$ is the expected operation cost when $\alpha^{\text {th }} \mathrm{PV}$ system is unavailable due to the unplanned failure. We assume it may happen with an equal probability across all days:

$$
\overline{D_{0} C_{u}^{(\alpha)}}=\frac{\sum_{d} \operatorname{DOC}\left(\mathrm{d}, X_{0_{\alpha}}^{(\alpha)}, \mathbb{E}\left[X^{(1)}, \ldots, X^{(\alpha-1)}, X^{(\alpha+1)}, \ldots, X^{(k)}\right]\right)}{N_{d}}
$$

Similarly, we can calculate the expected cost when $X^{(\alpha)}=$ $X_{i_{\alpha}}^{(\alpha)}$

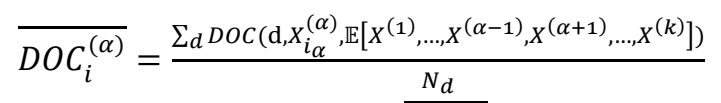

In the operation model, we use $\overline{D O C^{*}}$ as a benchmark. The penalty caused by preventive maintenance $C_{p}^{(\alpha)}$, unplanned failure $C_{u}^{(\alpha)}$ of $\alpha^{\text {th }} \mathrm{PV}$ system can be calculated equation (15) and (16) respectively.

$$
C_{p}^{(\alpha)}=\overline{D O C_{p}^{(\alpha)}}-\overline{D O C^{*}}
$$

$$
C_{u}^{(\alpha)}=\overline{D O C_{u}^{(\alpha)}}-\overline{D O C^{*}}
$$

The expected penalty caused by performance degradation due to only $i_{\alpha}$ arrays are functional can be calculated by $C_{i}^{(\alpha)}$.

$$
C_{v, i}^{(\alpha)}=\overline{D O C_{i}^{(\alpha)}}-\overline{D O C^{*}}
$$

One complication of calculating the equations (11)-(14) is the $\mathbb{E}\left[X^{(1)}, \ldots, X^{(\alpha-1)}, X^{(\alpha+1)}, \ldots, X^{(k)}\right]$ is unknown and affected by maintenance policies of all PV systems due to operation dependence. In the developed approach, we calculate the expected performance of all PV systems through iteration. To initialize the computation, we first assign the equal probability to all performance states of PV systems. Then, we calculate the steady state probabilities for each PV system at the optimal maintenance strategy. The steady state probabilities are then used to update the expected performance of all PV systems. The process iterates until the expected performance of all PV systems coverage. In the next section, we focus on describing the lower layer maintenance model and expressing with the expected performance of PV systems in term of steady state probabilities of PV system maintenance model.

\section{B. Lower layer (asset maintenance model)}

The lower layer model is to tackle the maintenance problem considering the operational information received from the upper layer. The PV system in the MG is indexed as hyperindex $\alpha$. The model is generalizable to apply to different types of multi-array PV system. Inspired by [14] and [15], we formulate the condition-based maintenance model with a continuous-time Markov chain. We model the failure of inverter as sudden failure and the malfunction of PV arrays as a performance degradation process of PV system. The state transition diagram for the condition-based maintenance is illustrated as Fig 4.

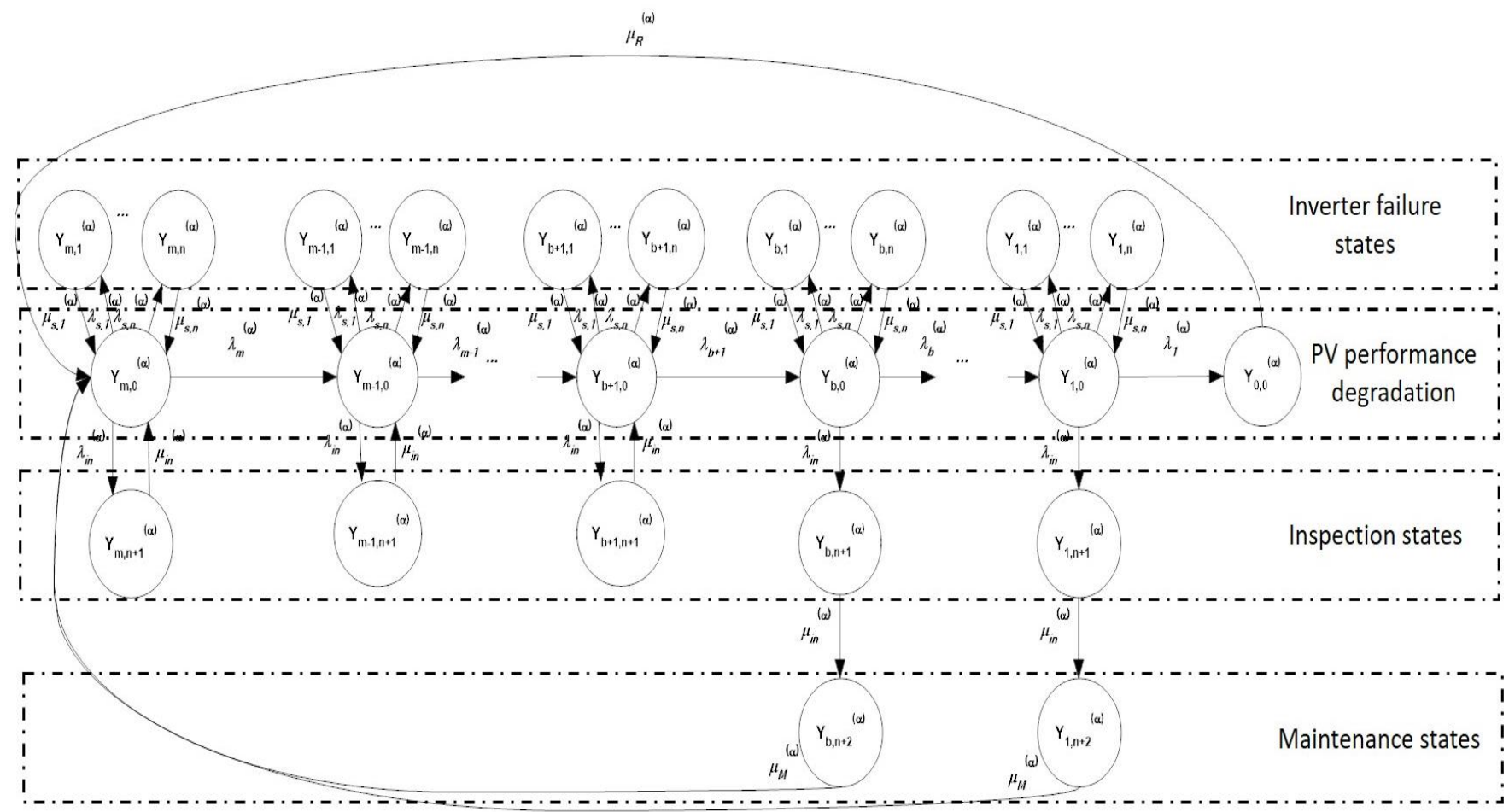

Fig. 4: The state transition diagram of PV system maintenance model 
In Fig. 4, the condition state of PV system is indicated as $Y_{i, j}^{(\alpha)}$. When $j=0$, it indicates the performance degradation of the PV system. $i$ is an index for the number of functioning PV arrays. For a PV system consisting $m$ arrays $(m>0, m \in \mathbb{N}), i=m$ represents that the $\mathrm{PV}$ is at as good as new condition. $i=0$ demonstrates that all arrays in the PV system are failed. The transition between state $Y_{i, 0}^{(\alpha)}$ to $Y_{i+1,0}^{(\alpha)}$ indicates the failure event of one array out of $i$ functioning arrays. We denote the transition rate as $\lambda_{i}^{(\alpha)}$. We assume the probability of more than one arrays fail simultaneously is negligible. In practice, the PV modules are much more reliable than inverters [18]. However, due to the large number of serially connected PV modules in a PV array and additive failure rate of the fuse in dc combiner, the failure rate of PV arrays is non-negligible [19]. The performance degradation of the PV system is modelled as a competing processes of PV arrays. We assume the failure rate of each array is identical and denoted as $\lambda_{d}^{(\alpha)}$, then $\lambda_{i}^{(\alpha)}=i \lambda_{d}^{(\alpha)}$. States with $1 \leq j \leq n$ indicate different inverter failure modes. The rate of $l^{\text {th }}$ failure mode is represented as $\lambda_{s, l}^{(\alpha)}$. We assume that all the inverter failures are self-announcing and disconnect the PV system from the grid; the duration for maintaining $l^{\text {th }}$ failure mode is denoted as $\mu_{s, l}^{(\alpha)}$. The PV's performance is assessed with a rate $\lambda_{i n}^{(\alpha)}$. The duration for the performance assessment is signified as $\mu_{i n}^{(\alpha)}$. If less than $b \mathrm{PV}$ arrays are functioning, the PV will be repaired to fully functional with a maintenance duration $\mu_{M}^{(\alpha)}$. If all PV arrays are failed, it will be replaced with a duration $\mu_{R}^{(\alpha)}$. The model is to determine the optimal threshold $b$ triggering the replacement of failed PV module in malfunctioned PV arrays. The analytical expression of steady state distribution for each state can be calculated through a list of equilibrium equations. All equilibrium equations could be formulated based on the concept that the sum of the input rates is identical to the sum of output rate at steady states. For the convenience of calculation, we first express all steady state probabilities in term of $\pi_{m, 0}^{(\alpha)}$ in equations $18-22$.

$$
\begin{gathered}
\pi_{i, 0}^{(\alpha)}=\left\{\begin{array}{cc}
\frac{\lambda_{m}^{(\alpha)}}{\lambda_{i}^{(\alpha)}} \pi_{m, 0}^{(\alpha)}, & i>b \\
\prod_{j=i}^{b-1} \frac{\lambda_{j+1}^{(\alpha)}}{\lambda_{j}^{(\alpha)}+\lambda_{i n}^{(\alpha)}} \frac{\lambda_{m}^{(\alpha)} \pi_{m, 0}^{(\alpha)}}{\lambda_{b}^{(\alpha)}+\lambda_{i n}^{(\alpha)}} & i \leq b
\end{array}\right. \\
\pi_{0,0}^{(\alpha)}=\frac{\lambda_{1}^{(\alpha)}}{\mu_{R}^{(\alpha)}} \prod_{j=1}^{b-1} \frac{\lambda_{j+1}^{(\alpha)}}{\lambda_{j}^{(\alpha)}+\lambda_{i n}^{(\alpha)}} \frac{\lambda_{m}^{(\alpha)} \pi_{m, 0}^{(\alpha)}+\lambda_{i n}^{(\alpha)}}{\pi_{i, l}^{(\alpha)}=}=\frac{\lambda_{s, l}^{(\alpha)}}{\mu_{s, l}^{(\alpha)}} \pi_{i, 0}^{(\alpha)} \\
\pi_{i, n+1}^{(\alpha)}=\frac{\lambda_{i n}^{(\alpha)}}{\mu_{i n}^{(\alpha)}} \pi_{i, 0}^{(\alpha)} \\
\pi_{i, n+2}^{(\alpha)}=\frac{\lambda_{i n}^{(\alpha)}}{\mu_{M}^{(\alpha)}} \pi_{i, 0}^{(\alpha)}
\end{gathered}
$$

Because the sum of all steady states probabilities is equal to probability 1 , we can calculate the $\pi_{m, 0}^{(\alpha)}$ as equation (23):

$$
\begin{aligned}
& =\sum_{i=b+1}^{\pi_{m, 0}^{(\alpha)}}\left(1+\sum_{l=1}^{m} \frac{\lambda_{s, l}^{(\alpha)}}{\mu_{s, l}^{(\alpha)}}+\frac{\lambda_{i n}^{(\alpha)}}{\mu_{i n}^{(\alpha)}}\right) \frac{\lambda_{m}^{(\alpha)}}{\lambda_{i}^{(\alpha)}} \\
& +\sum_{i=1}^{b} \prod_{j=i}^{b-1} \frac{\lambda_{j+1}^{(\alpha)}}{\lambda_{j}^{(\alpha)}+\lambda_{i n}^{(\alpha)}} \frac{1}{\lambda_{b}^{(\alpha)}+\lambda_{i n}^{(\alpha)}} \lambda_{m}^{(\alpha)}\left(1+\sum_{l=1}^{n} \frac{\lambda_{s, l}^{(\alpha)}}{\mu_{s, l}^{(\alpha)}}\right. \\
& \left.+\frac{\lambda_{i n}^{(\alpha)}}{\mu_{i n}^{(\alpha)}}+\frac{\lambda_{i n}^{(\alpha)}}{\mu_{M}^{(\alpha)}}\right) \\
& \left.+\frac{\lambda_{1}^{(\alpha)}}{\mu_{R}^{(\alpha)}} \prod_{j=1}^{b-1} \frac{\lambda_{j+1}^{(\alpha)}}{\lambda_{j}^{(\alpha)}+\lambda_{i n}^{(\alpha)}} \frac{1}{\lambda_{b}^{(\alpha)}+\lambda_{i n}^{(\alpha)}} \lambda_{m}^{(\alpha)}\right]^{-1}
\end{aligned}
$$

By combining the computed operation cost in (15) - (17) with the steady state information in (18) to (23), the overall cost for PV system can be calculated by Equation (24):

$$
\begin{aligned}
C_{S}^{(\alpha)}=\sum_{i=1}^{m} C_{v, i}^{(\alpha)} \pi_{i, 0}^{(\alpha)} & +C_{p}^{(\alpha)}\left(\sum_{i=1}^{m} \pi_{i, n+1}^{(\alpha)}+\sum_{i=1}^{b} \pi_{i, n+2}^{(\alpha)}\right) \\
& +C_{u}^{(\alpha)}\left(\sum_{i=1}^{m} \sum_{l=1}^{n} \pi_{i, l}^{(\alpha)}+\pi_{0,0}^{(\alpha)}\right) \\
& +C_{i n}^{(\alpha)} \sum_{i=1}^{m} \mu_{i n}^{(\alpha)} \pi_{i, n+1}^{(\alpha)} \\
& +C_{M}^{\prime(\alpha)} \sum_{i=1}^{b} \mu_{M}^{(\alpha)} \pi_{i, n+2}^{(\alpha)} \\
& +\sum_{i=1}^{m} \sum_{l=1}^{n^{\prime}} C_{s, l}^{(\alpha)} \mu_{s, l}^{(\alpha)} \pi_{i, l}^{(\alpha)} \\
& +C_{R}^{\prime(\alpha)} \mu_{R}^{(\alpha)} \pi_{0,0}^{(\alpha)}
\end{aligned}
$$

The overall cost for the $\alpha^{\text {th }} \mathrm{PV}$ system is the summation of penalty of performance degradation, downtime due to maintenance and failures, inspection cost, major maintenance cost, replacement cost. By comparing the $C_{S}^{(\alpha)}$ at different $b$ value, we can find the optimal maintenance threshold $b$ to minimize the $C_{S}^{(\alpha)}$. Then we can update the expected performance of $\alpha^{\text {th }} \mathrm{PV}$ system with equation. (25) and (26).

$$
\begin{gathered}
\mathbb{E}\left[X_{0 \alpha}^{(\alpha)}\right]=\pi_{0,0}^{(\alpha)}+\sum_{i=1}^{b} \pi_{i, n+2}^{(\alpha)}+\sum_{i=1}^{m} \sum_{j=1}^{n} \pi_{i, j}^{(\alpha)} \\
\mathbb{E}\left[X_{i \alpha}^{(\alpha)}: 0<i \leq m, i \in \mathbb{N}\right]=\pi_{i, 0}^{(\alpha)}+\pi_{i, n+1}^{(\alpha)}
\end{gathered}
$$

This process is applied to all PV systems and iterated until all $C_{S}^{(\alpha)}: 1 \leq \alpha \leq k$ reaching to convergence. Then, the expected annual ownership cost of the $\mathrm{MG} C_{G}$ can be calculated as equation (27).

$$
C_{G}=\overline{D O C^{*}}+\sum_{\alpha=1}^{k} C_{S}^{(\alpha)}
$$

\section{ILLUSTRATIVE CASE STUDY}

In this section, we demonstrate the applicability of the overall approach with an illustrative example. Consider a MG, as 
illustrated in Fig 1. Nodes D1 and D2 represent residential and commercial sectors, respectively. Two PV systems with rated capacities of, respectively, $300 \mathrm{~kW}$ and $1200 \mathrm{~kW}$ are denoted as nodes R1 and R2. Both R1 and R2 are multi-array PV systems with 5 and 20 arrays. 15 PV modules are considered in each array. The hourly output power in renewable nodes is determined according to hourly solar radiation. Three years' historical data on demand profiles, solar radiation, and electricity price are considered. Nodes S1 and S2 represent ESSs with $300 \mathrm{kWh} / 60 \mathrm{~kW}$ and $1600 \mathrm{kWh} / 220 \mathrm{~kW}$ (the first number is storage capacity and the second number indicates the maximum power capacity or power rating), which are determined according to [9]. Also, the following eligibility matrices show the configuration of the above network:

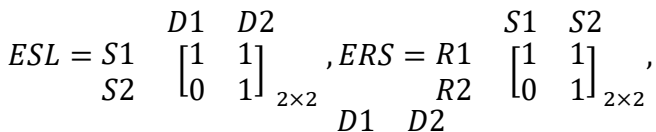

$$
\begin{aligned}
& E R L=R 1 \quad\left[\begin{array}{ll}
1 & 0 \\
0 & 1
\end{array}\right]_{2 \times 2}
\end{aligned}
$$

Table 1 shows the maintenance parameters and costs considered in this example (failure and maintenance rates are based on the real solar farm in a university campus in New Jersey. Cost values are adopted based on the study developed in [16]). According to this table, the maintenance duration is non-negligible (several days). Knowing the actual value of PV system in different days leads to better maintenance planning to avoid the high penalty cost of failure or performance degradation. As illustrated, maintenance action cost is a function of the number of modules that need to be replaced which is determined by the maintenance strategy.

\section{Table 1 - maintenance parameters and costs}

\begin{tabular}{cll}
\hline \hline Parameters & Value $(\boldsymbol{\alpha}=\mathbf{1}, \mathbf{R} 1)$ & Value $(\boldsymbol{\alpha}=\mathbf{2}, \mathbf{R} 2)$ \\
\hline$m^{(\boldsymbol{\alpha})}$ & 5 & 20 \\
$n^{(\boldsymbol{\alpha})}$ & 5 & 5 \\
$1 / \mu_{s, 1}^{(\boldsymbol{\alpha})}$ & 6 days & 6 days \\
$\lambda_{s, 1}^{(1)}$ & $0.5 /$ per year & $0.5 /$ per year \\
$C_{s, 1}^{\prime(\boldsymbol{\alpha})}$ & 2000 & 12000 \\
$1 / \mu_{s, 2}^{(\boldsymbol{\alpha})}$ & 4 days & 4 days \\
$\lambda_{s, 2}^{(\boldsymbol{\alpha})}$ & $0.3 /$ per year & $0.3 /$ per year \\
$C_{s, 2}^{(\boldsymbol{\alpha})}$ & $2000 \$$ & $12000 \$$ \\
$1 / \mu_{i n}^{(\boldsymbol{\alpha})}$ & 1 mins & 1 mins \\
$1 / \lambda_{i n}^{(\boldsymbol{\alpha})}$ & 1 day & 1 day \\
$C_{i n}^{\prime(1)}$ & 0 & 0 \\
$C_{R}^{\prime(\boldsymbol{\alpha})}$ & $360,000 \$$ & $1,440,000 \$$ \\
$1 / \mu_{R}^{(\boldsymbol{\alpha})}$ & 15 days & 15 days \\
$C_{M}^{\prime(\boldsymbol{\alpha})}$ & $3000+1920\left(\mathrm{~m}^{(1)}-\mathrm{b}^{(1)}\right)$ & $3000+1920\left(\mathrm{~m}^{(2)}-\mathrm{b}^{(2)}\right)$ \\
$1 / \mu_{M}^{(\boldsymbol{\alpha})}$ & 1 days & 1 days \\
\hline \hline
\end{tabular}

In the following section, we present the average annual operation cost of a MG, described in Fig 1, in different performance degradations and failure states of R1 and R2 (calculated in the upper layer). Then we present the optimal maintenance strategy for each of PV systems. Since the performance of PV systems is observable in real-time, the only decision variable in maintenance planning is determining the threshold state for major maintenance action (threshold state "b"). For comparative analysis, we run the top-down model for the MG without ESDs, and analyze the impact of ESDs on the MG's maintenance planning. The existence of ESDs in a MG increases the value of PV systems, so we expect that the existence of ESDs brings the threshold stage earlier (higher " $b$ " value).

As mentioned earlier R1 and R2, respectively, consist of 5 and $20 \mathrm{PV}$ arrays. Therefore, there exist 6 and 21 states of operation for R1 and R2. For example, renewable resource R1 is operating with $0 \%, 20 \%, 40 \%, 60 \%, 80 \%$ and $100 \%$ of its maximum capacity according to the number of functioning PV arrays. Fig. 5Fig. 5 shows the average annual operation cost of the example case when PV systems are operating in different states of deterioration.

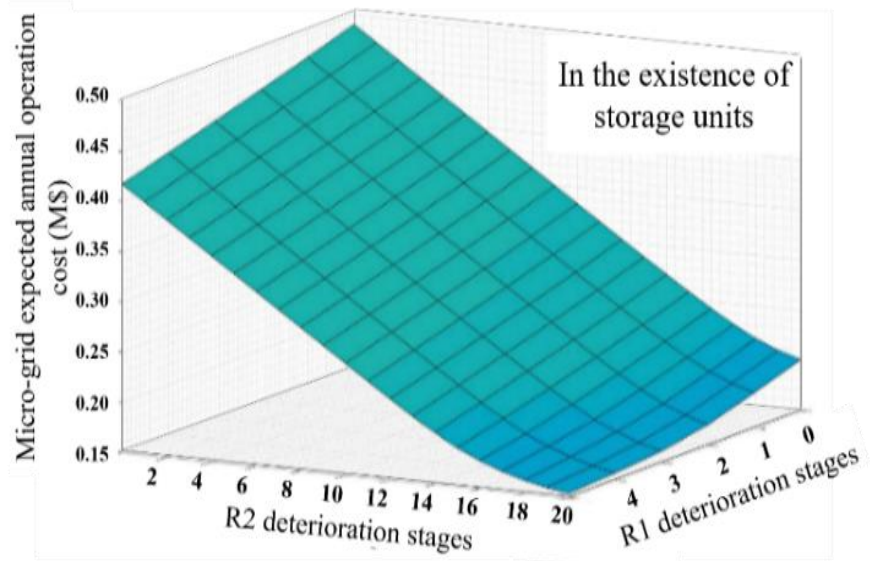

Fig. 5: MG average annual operation cost with different performances of $P V$ systems (in the existence of storage units)

In the lower layer, the optimal threshold for major maintenance action is determined with considering these operation cost values received from the upper layer. The maintenance model results show that the optimal threshold state "b" for renewables $\mathrm{R} 1$ and R2 are respectively 4 and 18 . It means that major maintenance action should be taken after $1^{\text {st }} \mathrm{PV}$ module failure in R1 and after $2^{\text {nd }} \mathrm{PV}$ module failure in R2. The optimal threshold state minimizes the average annual cost in the MG. It is worthwhile highlighting that the major novelty of the proposed model is that it is optimizing the long-term maintenance strategy of PV systems by considering the operational condition of the MG.

The value of the ESDs can be analyzed by comparative analysis. We consider the same MG in the previous example without any ESDs. In the absence of ESDs, the excessive output of renewable energy will be wasted. Hence, the value generated from PV systems decreases in the absence of storage units. Fig. 6Fig. 6 shows the MG's expected annual operating costs in the absence of storage units. As illustrated, the expected annual operation costs are close to each other in deterioration stages above 16 in $\mathrm{R} 2$ and 1 in R1. Thus, we expect that the maintenance model postpones the major maintenance action to the smaller threshold state " $\mathrm{b}$ " in the absence of storages. Running a maintenance model for the new operational condition of the network shows the same results. The maintenance model suggests doing a major maintenance action 
after $4^{\text {th }}$ PV module failure (threshold " $b$ ", 16) in R2 and after $3^{\text {rd }} \mathrm{PV}$ module failure (threshold " $\mathrm{b}$ ", 2 ) in R1.

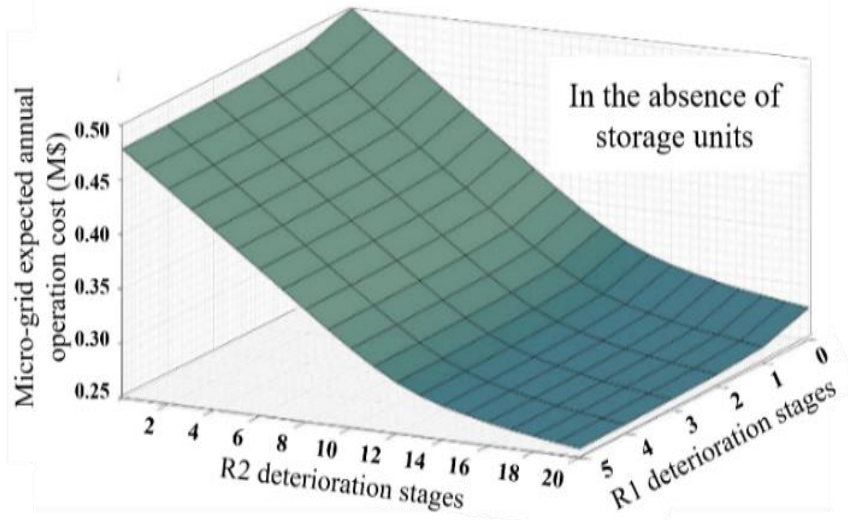

Fig. 6: MG average annual operation cost with different performances of $P V$ systems (in the absence of storage units)

Moreover, comparing the average annual total cost of a MG in these two examples (when optimal threshold "b" is selected) reveals the value that ESDs add to the PV systems in a MG. Fig. 7 demonstrates the average annual cost of a MGin these two examples for different values of threshold state "b". As illustrated in Fig.6 (c) and (d) the minimum ownership cost in the existence and absence of storage units are about $2.11 \times 10^{5} \$$ and $2.98 \times 10^{5} \$$ respectively. This implies that the existence of storage units approximately adds $8.7 \times 10^{4} \$$ to the value generated by PV system R2 in the MG.

The illustrative example shows that the maintenance strategy of PV systems should be optimized based on their value within the
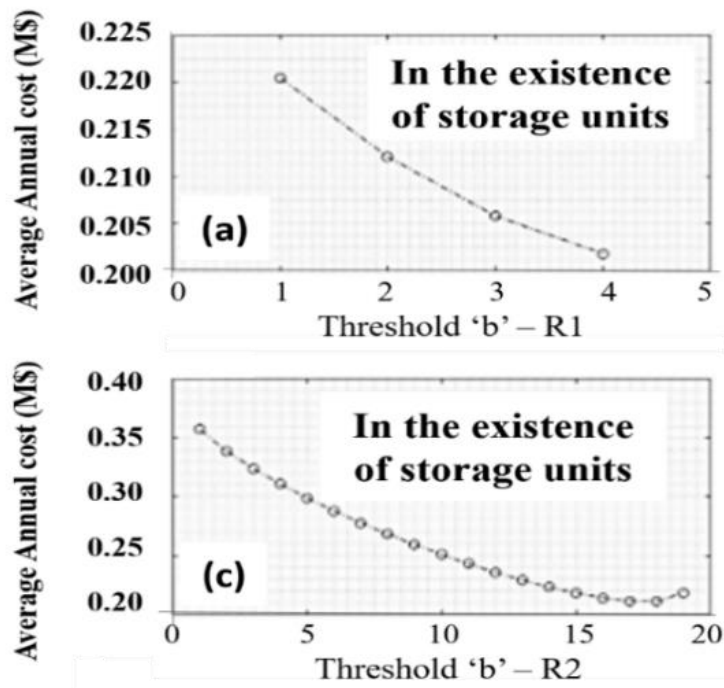

Fig. 7: Comparing average annual total cost for different threshold states " $b$ " of $R 1$ and $R 2$ in two examples

\section{CONCLUSION}

In this paper, we investigated the operation and maintenance policy for the grid-connected solar-powered MG composited by multi-array PV systems and ESDs. A top-down approach for optimizing the maintenance policies of PV systems is developed. In the upper layer, the maximum value of MG under different condition states of PV systems is calculated. This
MG. A PV system's value needs to be expressed by considering the operational condition of the network. By considering the network level information in asset level maintenance planning, it enables the network owner to plan the maintenance expenditure more efficiently.

PV systems are generally serviced by their manufacturers and the warranty contracts are stipulated between the service provider and the PV system owner. Under such contracts, all material cost for the replacement of system components are covered by the service provider for the duration of the warranty period. Moreover, system owner pays the service provider a fixed amount of money for the warranty period which is usually relative to the system capacity. This kind of service contract does not consider the real value of the PV system within the MG and only consider the system size, which may lead to waste of the money for either side. This study suggests that the warranty contract between service provider and system owner should be based on the performance of the system within the MG. For instance, our illustrative example shows that the value of the same capacity PV system (R2) is more in the existence of ESD, which means that system owner should spend more on maintenance to maintain the output of system over $90 \%$. However, in the absence of ESDs the owner should spend less on maintenance since $80 \%$ of performance is still economically beneficial. If the warranty contract between system owner and service provider is stipulated based on the system performance (meaning that system owner pays a percentage of electricity cost saved as a result of PV system operation to the service provider in exchange for the maintenance service), then it is mutually beneficial for both service provider and system owner with such type of warranty contract.
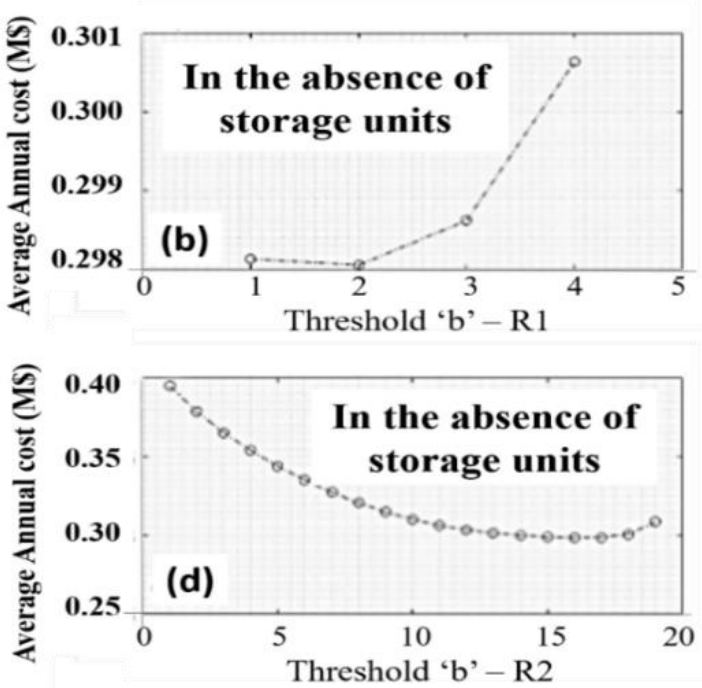

information is then utilized in the lower layer maintenance model. The long-term asset's ownership cost of the MG could be expressed analytically by disaggregating the network level information. It enables us to compare the performance of different maintenance policies and find the optimal strategy to minimize the network ownership cost. Presented case studies illustrate that same PV systems in MGs with a different configuration should have different maintenance strategies. The proposed approach could be used in the stipulation process 
between MG owner and PV system maintenance provider to minimize the money waste on both sides.

\section{ACKNOWLEDGMENT}

This research was partly funded by the EPSRC/Innovate UK Centre for Smart Infrastructure and Construction (EP/N021614/1) and also supported by Sustain-Owner (Sustainable Design and Management of Industrial Assets through Total Value and Cost of Ownership), a project sponsored by the EU Framework Programme Horizon 2020, MSCA-RISE-2014: Marie Skodowska-Curie Research and Innovation Staff Exchange (Rise) (grant agreement number 645733 Sustain-owner H2020-MSCA-RISE-2014).

\section{REFERENCES}

[1] Y. Kanoria, A. Montanari, D. Tse, and B. Zhang, "Distributed storage for intermittent energy sources:Control design and performance limits," in Communication, Control, and Computing (Allerton), 2011 49th Annual Allerton Conference on, 2011, pp. 1310-1317.

[2] R. Khalilpour and A. Vassallo, "Planning and operation scheduling of PV-battery systems: A novel methodology," Renew. Sustain. Energy Rev., vol. 53, pp. 194-208, 2016.

[3] H. T. Le and T. Q. Nguyen, "Sizing energy storage systems for wind power firming: An analytical approach and a costbenefit analysis," in Proc. Power Energy Soc. Gen. Meet., 2008, pp. 20-24.

[4] S. X. Chen, H. B. Gooi, and M. Wang, "Sizing of energy storage for microgrids," IEEE Trans. Smart Grid, vol. 3, no. 1, pp. 142-151, 2012.

[5] P. Harsha and M. Dahleh, "Optimal sizing of energy storage for efficient integration of renewable energy," in Decision and Control and European Control Conference (CDC-ECC), 2011 50th IEEE Conference on, 2011, pp. 5813-5819

[6] K. M. Chandy, S. H. Low, U. Topcu, and H. Xu, "A simple optimal power flow model with energy storage," in Decision and Control (CDC), 2010 49th IEEE Conference on, 2010, pp. 1051-1057.

[7] M. D. Hopkins, A. Pahwa, and T. Easton, "Intelligent dispatch for distributed renewable resources," IEEE Trans. Smart Grid, vol. 3, no. 2, pp. 1047-1054, 2012.

[8] J. Silvente, G. M. Kopanos, E. N. Pistikopoulos, and A.Espuña, "A rolling horizon optimization framework for the simultaneous energy supply and demand planning in microgrids," Appl. Energy, vol. 155, pp. 485-501, 2015.

[9] K. Mahani, F. Farzan and M.A. Jafari, "Network-aware approach for energy storage planning and control in the network with high penetration of renewables," Applied Energy, vol. 195, pp. 974-990, 2017.

[10] S. V. Dhople and A. D. Dominguez-Garcia, "Estimation of photovoltaic system reliability and performance metrics," IEEE Trans. Power Syst., vol. 27, no. 1, pp. 554-563, 2012.

[11] S. N. Kamenopoulos and T. Tsoutsos, "Assessment of the safe operation and maintenance of photovoltaic systems," Energy, vol. 93, Part 2, pp. 1633-1638, Dec. 2015.

[12] M. D. Hopkins, A. Pahwa, and T. Easton, "Intelligent dispatch for distributed renewable resources," IEEE Trans. Smart Grid, vol. 3, no. 2, pp. 1047-1054, 2012.

[13] T. Mukai, M. Tomasella, A. K. Parlikad, N. Abe, and Y. Ueda, "The Competitiveness of Continuous Monitoring of Residential PV Systems: A Model and Insights From the Japanese Market,” IEEE Trans. Sustain. Energy, vol. 5, no. 4, pp. 1176-1183, 2014.

[14] L. Bian and N. Gebraeel, "Stochastic framework for partially degradation systems with continuous component degradation-rate-interactions," Nav. Res. Logist. NRL, vol. 61, no. 4, pp. 286-303, 2014.

[15] D. Chen and K. S. Trivedi, "Closed-form analytical results for condition-based maintenance," Reliab. Eng. Syst. Saf., vol. 76, no. 1, pp. 43-51, Apr. 2002.

[16] J. Jacobi and R. Starkweather, "Solar Photovoltaic Plant Operating and Maintenance Costs," ScottMadden Rep. Sept., 2010.

[17] N. Moslemi, M. Kazemi, S. M. Abedi, H. KhatibzadehAzad, and M. Jafarian, "Maintenance Scheduling of Transmission Systems Considering Coordinated Outages," IEEE Syst. J., 2017.

[18] G. Petrone, G. Spagnuolo, R. Teodorescu, M. Veerachary, and M. Vitelli, 'Reliability issues in photovoltaic power processing systems', IEEE Trans. Ind. Electron., vol. 55, no. 7, pp. 2569-2580, 2008.

[19] P. S. Shenoy, K. A. Kim, B. B. Johnson, and P. T. Krein, 'Differential power processing for increased energy production and reliability of photovoltaic systems', IEEE Trans. Power Electron., vol. 28, no. 6, pp. 2968-2979, 2013.

[20] P. Zhang, Y. Wang, W. Xiao, and W. Li, 'Reliability evaluation of grid-connected photovoltaic power systems', IEEE Trans. Sustain. Energy, vol. 3, no. 3, pp. 379-389, 2012.

\section{BIOGRAPHIES}

Khashayar Mahani received his B.S. degree in Electrical and Control Engineering form University of Tehran, Tehran, Iran in 2011. He is currently a Ph.D. candidate in the Department of Industrial and System Engineering, Rutgers, the state university of New Jersey. He is also a member of the Laboratory on Energy Sustainability and Systems (LESS) Research Group, Rutgers University, which focuses on modeling, development and analysis of sustainable and smart energy solutions. His research interests are in Energy Storage management, Building Energy Management, Model Predictive Control and Network-Aware Planning \& Control.

Zhenglin Liang received the Ph.D. degree in reliability engineering and asset management from the University of Cambridge, U.K., in 2016. Currently, he is a research associate at the Distributed Information and Automation Laboratory at the University of Cambridge. His research interests include stochastic process, predictive maintenance, maintenance optimization and reliability of power equipment.

Ajith Kumar Parlikad received the Ph.D. degree in manufacturing management from Cambridge University, Cambridge, U.K., in 2006. He is now a Senior Lecturer in Industrial Systems with Cambridge University, Cambridge, U.K. His research interests include asset investment and maintenance decisionmaking, particularly based on value. His research has been funded by the EPSRC and industry. He sits on the Executive Committee in the Institution of Engineering and Technology TPN on Asset Management, U.K., and is the Chair of the Academic and Research Network for the Institute of Asset Management, U.K.

Mohsen Jafari (M'97) received the Ph.D. degree from Syracuse University in 1985. He has directed or co-directed a total of over 23 million U.S. dollars in funding from various government agencies, including the National Science Foundation, the Department of Energy, the Office of Naval Research, the Defense Logistics Agency, the NJ Department of Transportation, FHWA, DARPA, the NJ Department of Health and Senior Services, NYC MTA, and industry in automation, system optimization, data modeling, information systems, and cyber risk analysis. He actively collaborates with universities and research institutes abroad. He has also been Consultant to several Fortune 500 companies as well as local and state government agencies. He is currently a Professor and the Chair of Industrial \& Systems Engineering, Rutgers University-New Brunswick. His research applications extend to manufacturing, transportation, healthcare and energy systems. He is a member of the IIE. He received the IEEE Excellence Award in service and research. 